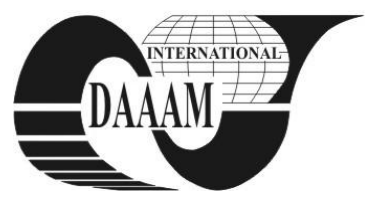

Annals of DAAAM for 2011 \& Proceedings of the 22nd International DAAAM Symposium, Volume 22, No. 1, ISSN 1726-9679 ISBN 978-3-901509-83-4, Editor B. Katalinic, Published by DAAAM International, Vienna, Austria, EU, 2011 Make Harmony between Technology and Nature, and Your Mind will Fly Free as a Bird

\title{
IMAGE OF THE ROUGHNESS SURFACE BY THE INTEGRALS CURVES
}

\author{
MACURA, D[usan]
}

Abstract: We conclude this section some applications of first order differential equations nonlinear. Differential equations describe many fundamental technological principles in engineering. The experimental dependences of the technological process have the differential equation. The solution of this equation is the analytical formule of the technological process. In this case it is the roughness of the worked describe by the integrale curves differential equation.

Key words: roughnes of the cut surface, differential equation, integral curves

\section{INTRODUCTION}

(9 pt, normal) Mathematical model of the engineering processes in various scientific branches is usually expressed by a differential equation or by a system of differential equations (Macurova, 2007). Nowadays, the issue of optimizing problems solution of the processes control lies most of all in the creation of the adequate mathematical model of a process (Balczak et al., 2010). The presented paper is aimed at the solution of the differential equation of the first order by the graphical method which represents an integrals curves (Macurova, 2010). Actually, the principal objective consists in searching for the solution that substitutes the solution of the original problem of the roughness surface.

\section{SOLUTION OF DIFFERENTIA EQUATION}

We considere the differential equation

$$
x=\frac{1}{2} t \cdot\left(x^{\prime}+\frac{4}{x^{\prime}}\right)
$$

Let $x=x(t) x^{\prime} \neq 0$, putting $x^{\prime}=p$, where $p$ is parameter, we obtain

$$
x=\frac{1}{2} t \cdot\left(p+\frac{4}{p}\right)
$$

This is a parametric notation of the equation (1) with dependent variable $t$. Differentiating (1) with respect to $t$, we have

$$
\begin{gathered}
x^{\prime}=\frac{1}{2}\left(p+\frac{4}{p}\right)+\frac{t}{2}\left(p^{\prime}-4 p^{-2} p^{\prime}\right) \\
p=\frac{1}{2} p+\frac{2}{p}+\frac{t}{2} p^{\prime} \cdot\left(1-4 p^{-2}\right) \\
\frac{p^{2}-4}{2 p}=\frac{t}{2} p^{\prime} \cdot\left(1-4 p^{-2}\right), p \neq 0 .
\end{gathered}
$$

If $p^{\prime}=0, p=c, c \in R-\{0\}$ then from equation we obtain $\frac{p^{2}-4}{2 p}=0$. The roots of this equation are $p \in\{-2,2\}$, and singular solutions are $x_{1}=2 t, x_{2}=-2 t$. Let $p^{\prime} \neq 0$. We consider that $t(p)$ is inverse function to $p(t)$. From we obtain

$$
\begin{aligned}
& \frac{p^{2}-4}{2 p}=\frac{t}{2} \frac{d p}{d t} \cdot\left(1-4 p^{-2}\right) \\
& \left(\frac{p^{2}-4}{2 p}\right) \frac{d t}{d p}-\frac{t}{2} \cdot\left(1-4 p^{-2}\right)=0 \\
& \left(\frac{p^{2}-4}{2 p}\right) t^{\prime}-\frac{t}{2} \cdot \frac{\left(p^{2}-4\right)}{p^{2}}=0 \\
& \frac{t^{\prime}}{t}=\frac{1}{p}, \\
& \int \frac{d t}{t}=\int \frac{d p}{p}+c, c \in R, t=c_{1} p, c_{1} \in R-\{0\}
\end{aligned}
$$

A parametric notation of independent variable is $t=c p$, where $c, p \in R-\{0\}$. From $t=c p$, and $x=\frac{1}{2} t \cdot\left(p+\frac{4}{p}\right)$, we obtain the general solution $t=c p$, and $x=\frac{1}{2} c\left(p^{2}+4\right)$, where $c, p \in R-\{0\}$.

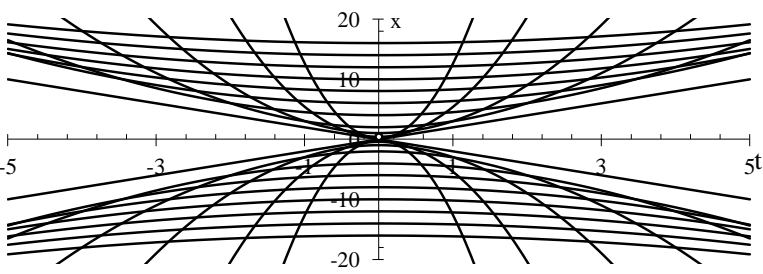

Fig. 1. Integrals curves of differential equation(1)

Let $f$ and $g$ are a continuous function in the interval $J$. A differential equation in the form

$$
x=f\left(x^{\prime}\right) t+g\left(x^{\prime}\right)
$$

is called Lagrange-d'Alembert's differential equation.

Letting $f\left(x^{\prime}\right)=x^{\prime}$ in (1) we obtain Clairaut's differential equation as a special form of Lagrange - d'Alembert's differential equation. A solution of Lagrange - d'Alembert's differential equation we obtain analogously as a solution of Clairaut's differential equation. Putting $x^{\prime}=p$, where $p$ parameter, we obtain $x=f(p) t+g(p)$ a parametric form 
of dependent variable. Differentiating with respect to $t$, we have

$$
\begin{aligned}
& x^{\prime}=f(p)+\frac{d f(p)}{d p} p^{\prime} t+\frac{d g(p)}{d p} \cdot p^{\prime} \\
& p=f(p)+\frac{d f(p)}{d p} p^{\prime} t+\frac{d g(p)}{d p} \cdot p^{\prime} \\
& p-f(p)=p^{\prime}\left(\frac{d f(p)}{d p} t+\frac{d g(p)}{d p}\right) .
\end{aligned}
$$

We consider two cases.If $p^{\prime}=0 \Rightarrow p=c, c \in R$, then from (2) we obtain $c-f(c)=0$ or $f(c)=c$. If the equation $f(c)=c$ has the roots $c_{i}, i \in\{1,2, \ldots, k\}$, then a functions $x=f\left(c_{i}\right) t+g\left(c_{i}\right), \quad i \in\{1,2, \ldots, k\}$ are the singular solutions of (1). Let $p^{\prime} \neq 0$. We derive a parametric form of independent variable. We consider that $t(p)$ is inverse function to $p(t)$. From (2) we obtain

$$
\begin{aligned}
& p-f(p)=\frac{d p(t)}{d t}\left(\frac{d f(p)}{d p} t+\frac{d g(p)}{d p}\right) \\
& (p-f(p)) \frac{d t(p)}{d p}-\frac{d f(p)}{d p} t=\frac{d g(p)}{d p} \\
& (p-f(p)) t^{\prime}-f^{\prime}(p) t=g^{\prime}(p) .
\end{aligned}
$$

This is a linear differential equation that we can solve for $t$

$$
t=c e^{\int \frac{f^{\prime}(p)}{p-f(p)} d p}+e^{\int \frac{f^{\prime}(p)}{p-f(p)} d p} \int \frac{g^{\prime}(p)}{p-f(p)} e^{\int \frac{f^{\prime}(p)}{f(p)-p} d p} d p
$$

The equation (3) is a parametric form, thus we get the general solution of d'Alembert's equation as a parametric representation $x=f(p)$.

$\left(c e^{\int \frac{f^{\prime}(p)}{p-f(p)} d p}+e^{\int \frac{f^{\prime}(p)}{p-f(p)} d p} \int \frac{g^{\prime}(p)}{p-f(p)} e^{\int \frac{f^{\prime}(p)}{f(p)-p} d p} d p\right)+g(p)$ $c \in R$

of the integral curves Fig. 1.

\section{CONCLUSION}

The paper presents method applied to the theory manufacturing engineering. Let the solution of the equation (1) is the roughness of the cut surface. Let a roughness is function dependences on the feed of the tool. The contribution of the lies in the consideration of the problem which is illustrations approached to by means of the functional (Hrubina et al 2009) analysis and the theoretical results obtained to create the background to the creation of algorithms applied to the solution of the defined problem. In general, we investigated the issue of the existence and unequivocalness of the solution $x(t)$ of the mathematical problem which substitutes the previous problem of the mathematical analysis, i.e. the differential equation of the first order. When assessing a mathematical model, a real phenomenon is simplified, schematized and the scheme obtained is expressed in dependence on the phenomenon complexity by means of a selected mathematical apparatus. A model has to reflect all most important factors affecting the process it has to provide a sufficiently true description of both quantitative and qualitative properties of the process that is being modeled. A mathematical description of the model structure according to the character of the process is a system of linear or non linear, differential or difference equations which reflect a mutual influence of different parameters. In the mathematical description of the equation they do not exclude the occurrence of equations of another type. Let the surface of the work machined with the feed $f$ with the tools radius $r_{\varepsilon}$. The dependency the roughness of the cutting speed and of the feed is predicted that the values of the maximum roughness by the relation( Kalpakijan, 1989)

$$
R z=\frac{f^{2}}{8 r_{\varepsilon}}
$$

It is possible to expressive the correlative betveen the described. The mathematical description of the dependency of the maximum roughness of the cut surface of the feed is possible formulated with the diferential equation and integral curves.Simulation of the mathematical model is a very powerful and videly used management technique for the analysis and study complex system surfaces. In previous we ere concerned with a formulation of models that could be solved analytically.Simulation may be defined as a technique that imitates the operation of a real world system as it evolves over time.A Available with most analytical models the simulation process involves executing or running the model through time usually on a computer, the real system with the results points. Mathematical modeling is not in contradiction to engineering modeling it is rather its replenishment. Engineering modeling is not determined to analyze specific properties of a mathematical description it is used to assess the objects adequacy based on the comparison of the values of some determining complexes in mathematical equations (Hrehova, 2005). With engineering modeling, the process is under investigation. Accordingly, various parameters of a mathematical and engineering model that is modeled on a computer are changed. This enables to obtain information about various variants of the investigated process very fast. Within a reasonable time it is possible to realize optimum variants of a model which means to carry out a mathematical model optimization. Mathematical and engineering modeling is much chapter than engineering modeling regardless it expresses money costs or time costs.

\section{REFERENCES}

Macurova, A. (2007). About solution of the non-linear differential equations, Technical University Kosice Publishing, ISBN 978-80-8073-910-2, Kosice

Balczak, S.; Hrubina, K.; Wessely, E. \& Macurova, A. (2010). Strategy of Manufacturing Programme Optimization in Manufacturing System, Annals of DAAAM for 2010 \& Proceedings of the $21^{\text {st }}$ International DAAAM Symposium, Vienna, ISSN 1726-9676, ISBN 978-3-901509-73-5, Katalinic, B. (Ed.), pp. 0563-0564, Published by DAAAM International, Vienna

Macurova, A. (2010). The roughness surface expressed by the mathematical model. Applied Surface Science Vol.256, No.18, (July 2010) page numbers (5591-5696), Elsevier, ISSN 0169-4332

Kalpakijan, S. (1989). Manufacturing Engineering and Technology. New York, Addison-Wesley Publisching Company, p.1199

Hrehova, S. (2009). Some statistics methods in technical education. Trends in education 2009. Olomouc, ISBN 97880-7220-316-1, p. 73-76. Published PU Olomouc. 